European Psychiatry

www.cambridge.org/epa

\title{
E-Poster Viewing
}

Anxiety disorders and somatoform disorders

\section{EPV0001}

Listening to music as a stress management tool

F. Lata and I. Kourtesis*

Department of Adult Psychiatry, Psychiatric Hospital of Athens "Dafni”, ATHENS, Greece

${ }^{*}$ Corresponding author.

doi: 10.1192/j.eurpsy.2021.1621

Introduction: The impact of listening to music on the stress response system has received increased attention lately.

Objectives: An update of the relative research.

Methods: Literature review.

Results: Listening to music seems to reduce stress by influencing both the hypothalamicpituitary-adrenal axis and the autonomous nervous system. Most studies, mainly conducted in healthy adults, show a decrease both in cortisol levels (a well-known stress-biomarker) and in sympathetic activity (reduction in heart rate frequency and blood pressure). Compositional elements of music such as melody, rhythm, tonality and frequency seem to influence individual relaxation responses. Most studies used classical music, nonetheless, the abovementioned effects were noticed irrespective of music genre. High-frequency music seems to have a greater role in stress-relief: $528 \mathrm{~Hz}$ frequency music apparently lowers cortisol and increases oxytocin (a modulator of stress response and social bonding) levels. A decrease in perceived anxiety and the induction of a positive mood state -particularly when relaxation was affirmed as the purpose of music listening- has been noticed using appropriate scales. Regarding particular settings, perioperative music may attenuate the neuroendocrine stress response caused by the surgery procedure. Music interventions in obstetric patients as well as in cardiovascular and cancer patients have led to similar findings. Finally, music appears to beneficially affect stress among patients with PSTD, fibromyalgia and depression.

Conclusions: The stress-relieving effect of music listening seems promising in clinical settings. The heterogeneity of the studies' sample and the "administration" of different music intervention "schemes" are among the main limitations of the current research.

Disclosure: No significant relationships.

Keywords: music listening; stress management

\section{EPV0002}

Monitoring anxiety symptoms with the monarca mobile application - an investigation of the usability during the first $\mathbf{1 8}$ months of commissioning in practice

K. Tarp ${ }^{1 \rtimes}$, T. Holmberg ${ }^{2}$, A.M. Møller ${ }^{3}$ and M. Lichtenstein ${ }^{4}$

${ }^{1}$ Research Unit For Telepsychiatry And E-mental Health, Centre For Telepsychiatry, Mental Health Services in the Region of Southern Denmark, Odense, Denmark; ${ }^{2}$ Research Unit For Telepsychiatry And Emental Health, Centre for Telepsychiatry, Odense C, Denmark; ${ }^{3}$ Health Promotion Research, Department Of Public Health, University of Southern Denmark, Odense, Denmark and ${ }^{4}$ Research Unit For Telepsychiatry And E-mental Health, Mental Health Services in the Region of Southern Denmark, Odense, Denmark

${ }^{*}$ Corresponding author. doi: 10.1192/j.eurpsy.2021.1622

Introduction: In cognitive behavioral treatment of anxiety disorders, registration of emotions and behavior is an important part of the intervention. Normally, paper and pencil is used but registrations on a mobile application such as MONARCA may be a useful alternative.

Objectives: This study investigates the usability of MONARCA during the first 18 months of commissioning in practice. 